\title{
Crescent Sign Following Enterprise-Stent assisted Coil Embolization of Distal Internal Carotid Artery Aneurysms
}

\author{
Young-Jin Kim, Je Young Yeon, Jong-Soo Kim, Seung-Chyul Hong \\ Department of Neurosurgery, Samsung Medical Center, Sungkyunkwan University School of Medicine, Seoul, Korea
}

\section{Received: August 28, 2019 \\ Accepted: September 10, 2019}

\section{Corresponding Author:}

Je Young Yeon, M.D., Ph.D.

Department of Neurosurgery, Samsung Medical Center, Sungkyunkwan University School of Medicine, 81 Irwon-ro, Gangnam-gu, Seoul 06351, Korea.

Tel: +82-2-2148-9878

Fax: +82-2-3410-0048

E-mail:yeonjay@hanmail.net

\begin{abstract}
Objective
We aimed to determine the "significant crescent signs" associated with delayed thromboembolic events (DTE) after changes in antiplatelet medication and to evaluate their incidence and natural course.
\end{abstract}

\section{Methods}

Between April 2009 and April 2011, 105 consecutive patients underwent stent-assisted coil embolization for unruptured intracranial aneurysms $(n=107)$ in a single tertiary hospital. Sixty-three cases fulfilled the inclusion criteria and were included in this study. Crescent sign was evaluated through follow-up magnetic resonance angiography (MRA) immediately (<48 hours), 6 to 12 months, 1 to 2 years, and 2 to 4 years and correlated with the occurrence of DTE.

\section{Results}

Crescent signs were identified in $26(41.3 \%)$ of 63 cases on immediate follow-up MRA. During the mean radiological follow-up of 53 months, the crescent signs (larger than $5 \mathrm{~mm}$ in length) persisted for more than 1 year in $11(42.3 \%)$ of 26 cases. The crescent signs on follow-up MRA showed decreased size in 10 of 26 cases and disappeared in 5 cases. DTE occurred in 6 (9.5\%) of 63 cases. In these 6 cases, all DTE occurred when the antiplatelet regimen was changed or stopped. Five of these six cases exhibited a crescent sign measuring over $5 \mathrm{~mm}$ in length, at the time of stroke.

\section{Conclusions}

Crescent signs may be associated with DTE. When crescent signs are larger than $5 \mathrm{~mm}$ in length and continuously identified on follow-up MRA, continued dual antiplatelet treatment is required.

Keywords: Intracranial Aneurysm; Self Expandable Metal Stent; Therapeutic Embolization; Antiplatelet Drugs

\section{INTRODUCTION}

Stent-assisted coiling has broadened the scope of endovascular aneurysm management in the past decade ${ }^{1-3)}$. Stent-assisted coiling has showed a favorable occlusion rate of aneurysms previously considered uncoilable with protection of the parent vessel ${ }^{1,3,4)}$. The

Copyright (C) 2019 The Korean Neurointensive Care Society

This is an Open Access article distributed under the terms of the Creative Commons Attribution Non-Commercial License (http://creativecommons.org/licenses/by-nc/4.0/) which permits unrestricted non-commercial use, distribution, and reproduction in any medium, provided the original work is properly cited. 
Enterprise Stent (Cordis Neurovascular, Miami, Fl.) is a widely used self-expandable, closed-cell type and represents a significant improvement in stent-assisted coiling of wide neck aneurysms.

However, despite development of stent-assisted procedures, incomplete stent apposition (ISA) and interruption of antiplatelet therapy can increase the risk of thromboembolic complications ${ }^{5,6)}$. Previous studies have reported that periprocedural thromboembolic complications after Enterprise stent-assisted embolization may be the result of ISA that was associated with the use of a stent in a tortuous parent vessel. The shape of an orphaned area of semilunar flow created by ISA is visible on 3T MRA and looks like a crescent, which is why they are referred to as "crescent signs." The crescent signs appear to be a risk factor for delayed thromboembolic events (DTE) and resolve spontaneously in only a small minority of cases $^{6-8)}$.

The optimal antiplatelet therapy protocol for prevention of thromboembolic complications after stent-assisted embolization of cerebral aneurysm has not been established. However, few studies reported that DTE were associated with cessation of antiplatelet therapy ${ }^{5,9,10)}$.

The objectives of this study were to identify "significant crescent signs" associated with DTE after changes in antiplatelet medication and to evaluate their incidence and natural course.

\section{METHODS}

\section{Patient selection}

There were 105 consecutive patients with 107 aneurysms treated with Enterprise stent-assisted embolization for intracranial aneurysms at our institution between April 2009 and April 2011. Patients were included in this study if they met the following criteria: 1) immediate ( $<48 \mathrm{hrs}$ ) and follow-up MRA was performed; 2) distal internal carotid artery (ICA) aneurysms were present; and 3) after discharge, the patients received antiplatelet medication. Seventy-five cases met the inclusion criteria. Exclusion criteria were as follows: 1$)$ use of multiple Enterprise stents $(n=5) ; 2)$ cavernous ICA aneurysm ( $n=2)$; and 3 ) clinico-radiological follow-up shorter than 6 months after embolization $(n=5)$. Finally, a total of 63 cases were eligible for this study. Patients' age and sex and the location and size (aspect and dome-to-neck ratio) of the aneurysm were analyzed in addition to comorbid conditions including hypertension, diabetes mellitus, hyperlipidemia, coronary artery disease, prior stroke history, and smoking history.

\section{Image assessment and follow-up}

During the admission period, all patients underwent brain MRI and MRA within 48 hours after the procedure. In the out-patient unit, all patients were evaluated with a follow-up MRA between 6 and 12 months, 1 and 2 years, and 2 and 4 years. The crescent sign was measured by measuring the length of the gap between the stent and vessel wall on immediate MRA and follow-up images (Fig. 1).

A DTE was defined as a symptomatic newly developed infarction on MRI more than 3 months after treatment, which involved vascular territory distal to the stented segment in the absence of other possible sources of thromboembolism.

\section{Antiplatelet management}

All patients received dual-antiplatelet therapy (DAT) consisting of aspirin (100 mg/day) and clopidogrel (75 mg/day for 7 days) before the procedure. After the procedure, all patients were maintained on DAT for a few months, followed by mono-antiplatelet therapy.

\section{Statistical analysis}

All analyses were performed using SPSS 20.0 version. Means and standard deviations were provided for continuous variables. Student's t-tests and Fisher's exact tests were used for analysis of the variables. A p-value of $<0.05$ was considered statistically significant.

\section{RESULTS}

Among the 105 patients (107 aneurysms), 63 cases were treated using Enterprise stent-assisted coil embolization and were included in this study. The patients consisted of 51 women (81\%, $55.7 \pm 7.4$ years) and 12 men ( $19 \%, 57.6 \pm 10.2$ years). Thirty-one patients' aneurysms were located on the paraclinoid segment (49.2\%), while 22 (34.9\%) aneurysms were located on the dorsal wall of the ICA. Aneurysms on the origin of the posterior communicating artery were found in 7 cases $(11.1 \%)$. Each aneurysm (1.6\%) was located on the origin of the ophthalmic and anterior choroidal artery or distal ICA (superior to anterior choroidal artery). The mean aspect ratio (height/neck) of aneurysms was 1.3 (range: 0.6 to 3.1 ) and the mean "dome to neck" ratio was 1.2 (range: 0.7 to 2.3 ). The locations of the treated aneurysms are listed in Table 1.

\section{Onset of DTE and antiplatelet status}

During the follow-up period, DTE occurred in 6 (9.5\%) of 63 cases (median: 10 months, range: 5 to 14 months). All 6 cases developed DTE in association with antiplatelet medication changes. Three of the six cases occurred in patients who were not on antiplatelet therapy (temporarily stopped for surgery, Fig. 2), and the 

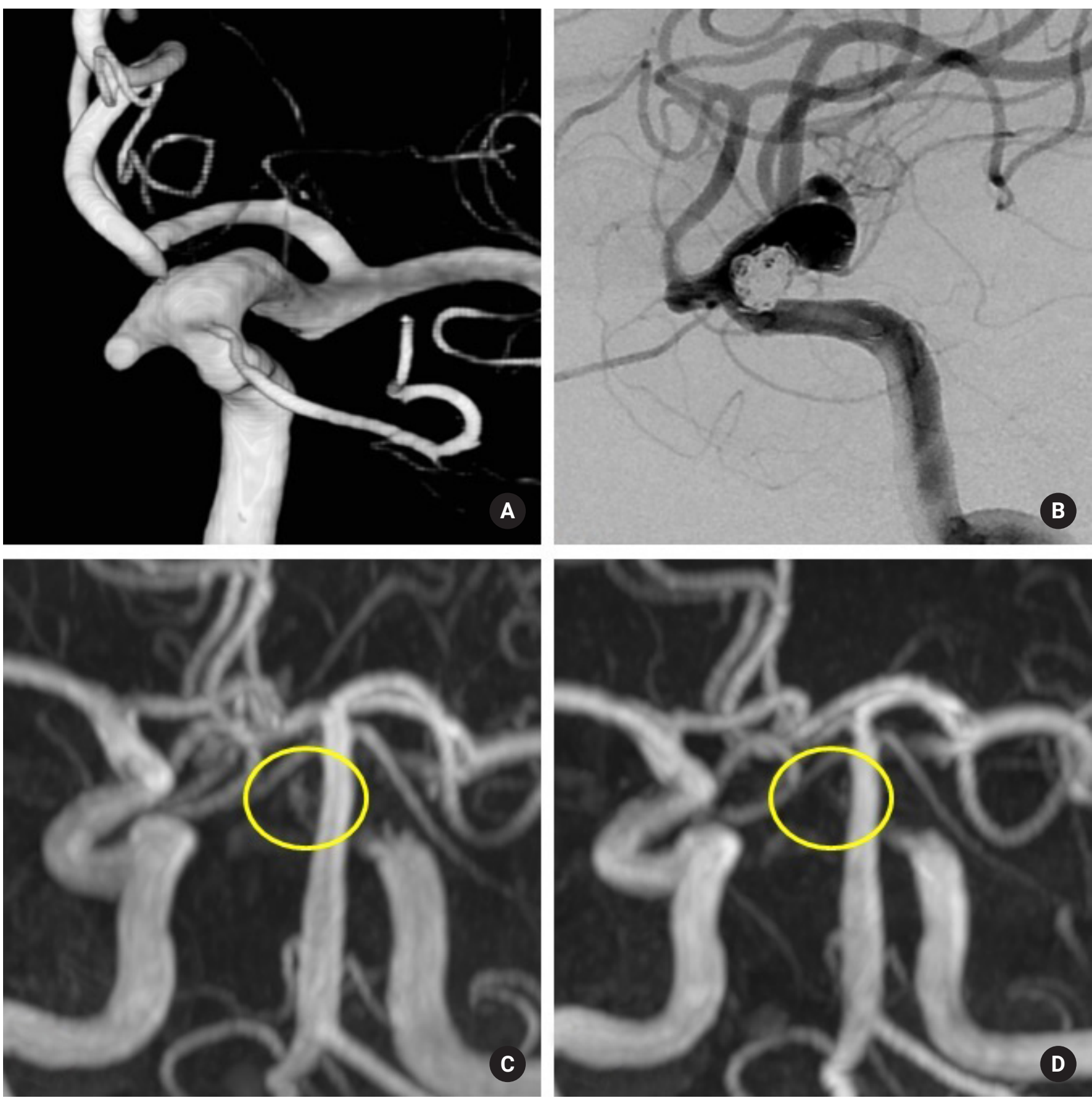

Fig. 1. A 65-year-old woman with a wide-neck left paraclinoid aneurysm. A: 3D-AP reconstructed image of aneurysm. B: DSA (digital subtraction angiography) after stent-assisted coil embolization. C: Immediate post-treatment MRA shows a crescent sign measuring $7 \mathrm{~mm}$ in length on the inner vessel curve (yellow circle). D: The crescent sign is not present on the follow-up MRA performed 1 year after treatment (yellow circle).

other 3 events occurred in patients who changed DAT to mono-antiplatelet therapy (MAT). The characteristics of patients with DTE are listed in Table 2.

\section{Correlation between patients' factors and DTE occurrence}

We also analyzed patient factors between those who experienced DTE and the remaining patients. Baseline characteristics are compared in Table 3. The incidence of DTE was higher in patients with a history of prior intracranial hemorrhage or infarction $(\mathrm{p}=0.002)$. There was no significant difference in the other patient clinical factors: age $(\mathrm{p}=0.144)$, hypertension $(\mathrm{p}=0.372)$, diabetes mellitus 
Table 1. Locations of treated aneurysms

\begin{tabular}{lc}
\hline Location & No. of Aneurysms (\%) \\
\hline ICA bifurcation & $0(0 \%)$ \\
Distal ICA (superior to anterior choroidal artery) & $1(1.6 \%)$ \\
Dorsal wall & $22(34.9 \%)$ \\
Anterior choroidal segment & $1(1.6 \%)$ \\
Posterior communicating artery & $7(11.1 \%)$ \\
Ophthalmic artery & $1(1.6 \%)$ \\
Paraclinoid segment & $31(49.2 \%)$
\end{tabular}

$(\mathrm{p}=0.182)$, hyperlipidemia $(\mathrm{p}=1.000)$, smoking history $(\mathrm{p}=0.404)$, and coronary artery disease $(\mathrm{p}=1.000)$. In the DTE group, 5 cases exhibited a crescent sign measuring over $5 \mathrm{~mm}$ in length at the time of stroke. Thus, we hypothesized that crescent signs over $5 \mathrm{~mm}$ may have clinical implications associated with DTE, and we evaluated the incidence of DTE between groups based on crescent signs measuring over $5 \mathrm{~mm}$. The results indicated that a persistent crescent sign measuring over $5 \mathrm{~mm}$, which we named as a "significant crescent sign," has a strong correlation with DTE occurrence $(\mathrm{p}=0.001)$.
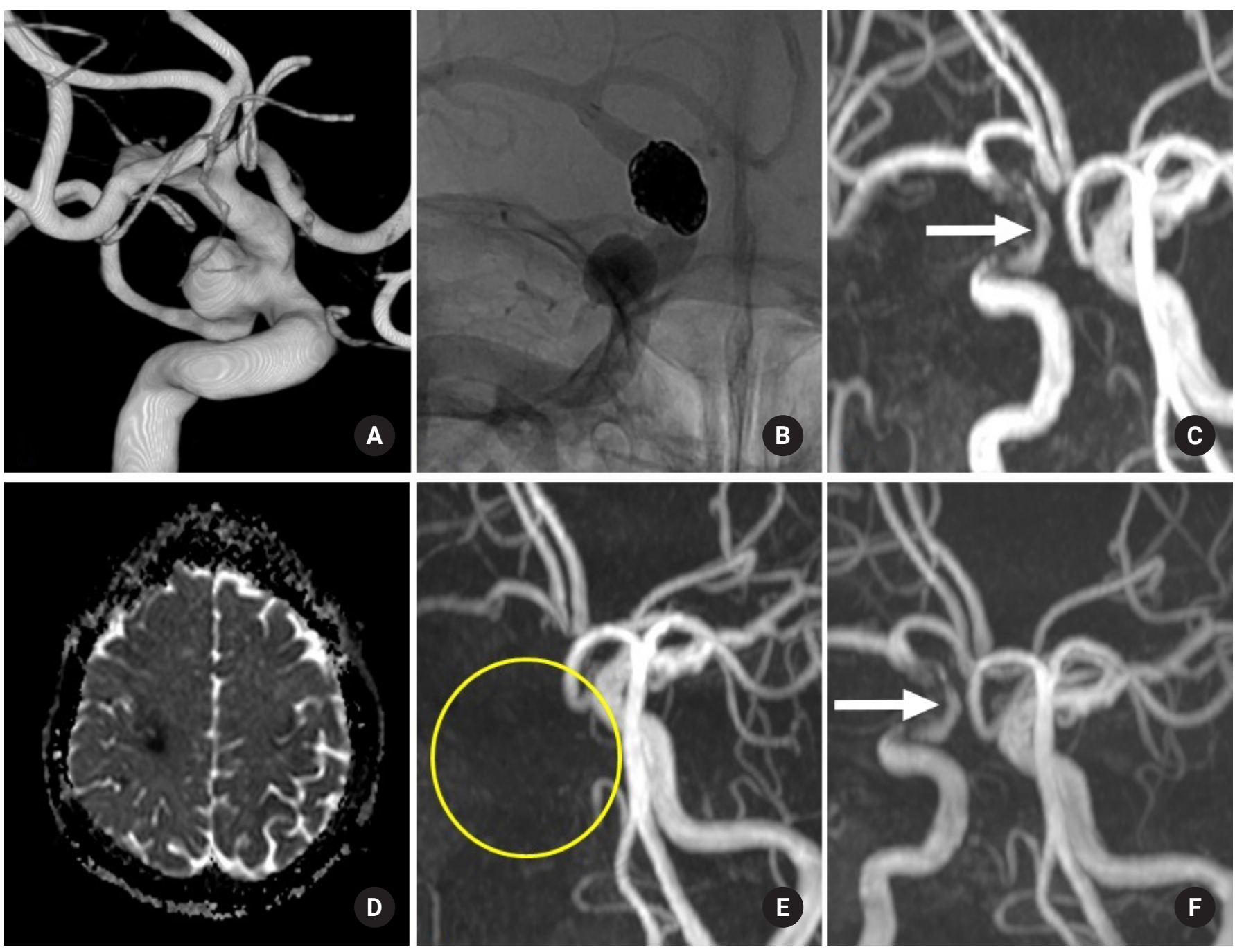

Fig. 2. A 55-year-old woman with a right posterior communicating artery aneurysm. Immediate post-procedural MRA reveals a crescent sign measuring about $12 \mathrm{~mm}$ on the medial aspect of the Enterprise stent. The patient received DAT (aspirin + clopidogrel), and brain MRA performed at 12 months after the procedure shows the same size of the crescent sign. The patient discontinued clopidogrel 5 days for knee arthroplasty at 14 months after embolization. After knee arthroplasty, the patient developed delayed thromboembolic events (DTE) (mild dysarthria and left arm weakness). A: Pre-procedural 3D reconstructed image of the aneurysm. B: DSA after stent-assisted coil embolization. C: MRA performed at 12 months after the procedure shows the crescent sign measuring $12 \mathrm{~mm}$ (white arrow). D: Acute infarction in the right hemisphere (apparent diffusion coefficient map obtained at the time of occurrence of DTE). E: MRA shows complete occlusion of the right ICA (yellow circle). F: The crescent sign persists on the MRA performed 5 years after the DTE (white arrow). 
Table 2. Characteristics of patients with ischemic complication after change in antiplatelet regimen

\begin{tabular}{|c|c|c|c|c|}
\hline No. & Age (yr), Sex & Aneurysm Location & $\begin{array}{l}\text { Time interval to } \\
\text { Event (month) }\end{array}$ & Antiplatelet medication \\
\hline 1 & $57 / \mathrm{F}$ & Paraclinoid & 10 & Aspirin+Clopidogrel for 9 months $\rightarrow$ Aspirin only for 1 month $\rightarrow$ Stroke \\
\hline 2 & $64 / \mathrm{F}$ & Paraclinoid & 11 & Aspirin + Clopidogrel for 7 months $\rightarrow$ Clopidogrel only for 4 months $\rightarrow$ Stroke \\
\hline 3 & $69 / \mathrm{M}$ & Paraclinoid & 14 & Aspirin + Clopidogrel for 4 months $\rightarrow$ Clopidogrel only for 10 months $\rightarrow$ Stroke \\
\hline 4 & $55 / \mathrm{F}$ & $\mathrm{PCoA}^{*}$ & 14 & $\begin{array}{l}\text { Aspirin }+ \text { Clopidogrel for } 1 \text { month } \rightarrow \text { Clopidogrel only for } 13 \text { months } \rightarrow \text { Temporary stop } \\
\text { for } 5 \text { days (knee arthroplasty) } \rightarrow \text { Stroke }\end{array}$ \\
\hline 5 & $57 / \mathrm{F}$ & Dorsal wall, Lt. ICA & 9 & $\begin{array}{l}\text { Aspirin }+ \text { Clopidogrel for } 8 \text { months à Clopidogrel only for } 1 \text { months } \rightarrow \text { Temporary stop } \\
\text { for } 5 \text { days (Lung cancer) } \rightarrow \text { Stroke }\end{array}$ \\
\hline 6 & $57 / \mathrm{F}$ & Dorsal wall, Rt. ICA & 5 & $\begin{array}{l}\text { Aspirin }+ \text { Clopidogrel for } 4 \text { months à Clopidogrel only for } 1 \text { month } \rightarrow \text { Temporary stop } \\
\text { for } 5 \text { days (Lung cancer) } \rightarrow \text { Stroke }\end{array}$ \\
\hline
\end{tabular}

No. 5 and 6 indicate both sides of the same patient.

Table 3. Univariate analysis of clinical chracteristics in two groups (DTE vs. Non-DTE)

\begin{tabular}{lccc}
\hline & DTE $(\mathrm{n}=6)$ & Non DTE $(\mathrm{n}=57)$ & $\mathrm{p}$-value \\
\hline Age & $59.8 \pm 7.9$ & $55.6 \pm 8.1$ & 0.144 \\
Hypertension & 3 & 17 & 0.372 \\
Diabetes mellitus & 1 & 1 & 0.182 \\
Hyperlipidemia & 1 & 9 & 1.000 \\
Smoking history & 1 & 4 & 0.404 \\
Prior stroke history & 3 & 1 & 0.002 \\
Coronary artery disease & 0 & 3 & 1.000 \\
Crescent sign $>5$ mm & 5 & 6 & 0.001 \\
\hline
\end{tabular}

DTE: delayed thromboembolic events.

Table 4. Correlation between the crescent sign and DTE occurrence with regard to change in antiplatelet regimen

$\begin{aligned} & \text { 1. Dual } \rightarrow \text { mono antiplatelet switch }(\mathrm{n}=61) \\ & \text { Significant crescent sign }>5 \mathrm{~mm}(\mathrm{n}=17)\end{aligned}$
No/minimal crescent sign $<5 \mathrm{~mm}(\mathrm{n}=44)$
$\begin{aligned} & \text { 2. Temporary medication stop }(\mathrm{n}=13) \\ & \text { Significant crescent sign }>5 \mathrm{~mm}(\mathrm{n}=3)\end{aligned}$
$\begin{aligned} & \text { No/minimal crescent sign }<5 \mathrm{~mm}(\mathrm{n}=10) \\ & \text { DTE: delayed thromboembolic events. }\end{aligned}$
$\begin{aligned} & \text { Incidence and natural course of the crescent sign and } \\ & \text { antiplatelet medication }\end{aligned}$

Crescent signs measuring over $5 \mathrm{~mm}$ were identified in 26 (41.2\%) of 63 cases on immediate post-procedural MRA $(<48$ hrs). The locations of crescent signs were the anterior genu of the carotid siphon $(n=21)$, between the ophthalmic artery and communicating segment $(n=3)$, neck remnant of aneurysm $(n=1)$, and untreated aneurysm within stent $(n=1)$. During the mean radiological follow-up of 53 months, crescent signs measuring over 5 $\mathrm{mm}$ persisted for 1 to 6 years in 11 (42.3\%) of the 26 cases. In 10 of the remaining 15 cases, the crescent signs were smaller in size (persistent minimal crescent). In the other 5 cases, the crescent signs disappeared or were not detectable on follow-up MRA.

In a total of 63 cases, patients had varied duration of DAT with an average of 6.1 months (range: 1 to 32 months). We analyzed the correlation between a significant crescent sign and incidence of DTE when antiplatelet therapy was changed or stopped (Table 4). If a crescent sign measuring over $5 \mathrm{~mm}$ existed at the time of changing antiplatelet regimen (DAT to MAT), the incidence of DTE was significantly increased compared to the group without crescent signs or that having minimal crescent signs $<5 \mathrm{~mm}$ $(p=0.005)$. When the patient stopped antiplatelet therapy tempo- 
rarily for any other reasons, if the patient had a significant crescent sign, the incidence of DTE was also increased compared to other groups $(\mathrm{p}=0.003)$.

\section{DISCUSSION}

The development of intracranial self-expanding stents has broadened the applicable range of endovascular treatment to intracranial aneurysms that are wide-necked, large, or giant-sized. The purpose of using a stent in embolization is prevention of coil protrusion in addition to a diversion effect of the hemodynamic blood flow to diminish intra-aneurysmal flow ${ }^{1,3,4)}$.

The Enterprise stent was introduced in May 2007. Recently, several studies of short- and long-term results for the Enterprise stent were reported, demonstrating excellent occlusion rate and device navigability, as well as low morbidity and mortality rates ${ }^{2,4,11)}$. Especially, a long-term report showed that $81 \%$ of angiograms demonstrated greater than $90 \%$ aneurysm occlusion rates and only $8.3 \%$ required retreatment. These results are similar to data reported in a large series of aneurysm coil embolization with and without stent assistance ${ }^{11)}$.

However, concern remains over the deployment of potentially thrombogenic sources into the intracerebral artery when ISA exists after stent-assisted coiling. Although ISA has been more reported in cardiology literature ${ }^{12-14)}$, recently, many studies have reported an association between ISA and ischemic events in the neurosurgical fields. The presence of ISA can be detected by its characteristic shape on MRA: a semilunar orphaned area of ICA, which looks like a crescent $\operatorname{sign}^{5)}$.

Heller et al. reported that in ISA of the Enterprise stent-mediated coiling of aneurysms of 50 patients, delayed ischemic events occurred in $8(16 \%)$ of 50 cases, and all cases involved patients with ISA ${ }^{6}$. In our study, DTE occurred in 6 cases (9.5\%) of 63 cases, and 5 of 6 cases had a significant crescent sign at the time of the event. The significant crescent sign was also statistically associated with DTE $(\mathrm{p}=0.001)$. Thus, we suggest that significant crescent signs (over 5 $\mathrm{mm}$ ) are an important factor in predicting the probability of DTE.

Considering the natural course of crescent signs, some crescent signs were observed consistently on follow-up images. Heller et al. ${ }^{6)}$ reported that ISA was identified in postoperative imaging studies in $22(44 \%)$ of 50 patients, with 19 (86\%) of 22 crescent signs persisting and 3 (14\%) of 22 crescent signs resolving on subsequent serial imaging. In this study, initially, significant crescent signs were identified in 26 (41.2\%) of 63 cases, and 11 (42.3\%) of the 26 cases showed persistent significant crescent signs on their last follow-up MRA. Resolution of the crescent sign on serial imaging could be explained by 3 possibilities as follows: 1 ) in-stent stenosis or thrombosis of the orphaned lumen; 2 ) delayed stent mi- gration; and 3) angle remodeling of a deployed stent ${ }^{(6)}$. In Heller et al's and our studies, however, only small crescent signs were resolved spontaneously. Considering that the crescent sign and incidence of DTE have a significant correlation, continuous follow-up seems necessary once the crescent sign is identified after stent-assisted coiling.

The post-procedural antiplatelet therapy has been accepted as the standard of care for prophylaxis against in-stent stenosis or thromboembolic events. Yamada et al. ${ }^{10)}$ reported that the use of DAT with aspirin and clopidogrel for elective coil embolization of unruptured cerebral aneurysms has been shown to reduce the rate of symptomatic thromboembolic complications. Mocco et al. ${ }^{5)}$ reported that all 7 (3\%) DTE cases were concomitant to cessation of DAT. Rossen et al. ${ }^{9)}$ reviewed 154 patients who underwent aneurysm treatment with stent techniques; despite all patients taking aspirin at the time of the ischemic events, $6(5 \%)$ ischemic events developed after clopidogrel discontinuation. In our study, 6 (9.5\%) of 63 cases experienced ischemic events and all 6 cases developed DTE in association with antiplatelet medication changes ( 3 casesMAT change, 3 cases-stopped temporarily). Regarding our results, especially in patients with a persistent significant crescent sign on follow-up MRA, changing the regimen or cessation of antiplatelet therapy could increase the risk of ischemic events. However, there remain questions regarding the duration of dual-antiplatelet therapy and the optimal time to switch to MAT. In the cardiovascular field, increasing thromboembolic events related to a drug-eluting stent after cessation of clopidogrel have led to the recommendation to maintain the antiplatelet therapy for 6 to 12 months ${ }^{15,16)}$. A retrospective study of 395 patients with 403 intracranial aneurysms treated with stent-assisted coil placement reports that longer-term dual antiplatelet therapy for more than 9 months and a late switch to mono-antiplatelet therapy were found to significantly reduce delayed ischemic stroke ${ }^{17)}$. To establish the optimal duration and method of antiplatelet therapy for the prevention of in-stent stenosis in patients with intracranial stents, especially when the ISA is present, further research is needed.

\section{Limitation}

As this study was a retrospective analysis, there is a possibility of selection bias. A relatively small number of patients compared with previous studies may be another limitation. The crescent sign was measured only in length in this study. We believe that various useful methods can be employed to evaluate the crescent sign.

\section{CONCLUSION}

After stent-assisted embolization, the crescent sign showed 
strong association with the incidence of DTE. Crescent signs measuring over $5 \mathrm{~mm}$ in length may have more important clinical implications. Antiplatelet strategies need to be more customized after Enterprise stent-assisted embolization due to an increased risk of developing DTE, especially with the presence of persistent significant crescent signs. Further research is warranted to determine the optimal antiplatelet regimen and duration in patients who undergo intracranial stent-assisted embolization with ISA.

\section{NOTES}

\section{Conflict of interest}

No potential conflict of interest relevant to this article was reported.

\section{REFERENCES}

1. Akpek S, Arat A, Morsi H, Klucznick RP, Strother CM, Mawad ME. Self-expandable stent-assisted coiling of wide-necked intracranial aneurysms: a single-center experience. AJNR Am J Neuroradiol 2005;26:1223-1231.

2. Higashida RT, Halbach VV, Dowd CF, Juravsky L, Meagher S. Initial clinical experience with a new self-expanding nitinol stent for the treatment of intracranial cerebral aneurysms: the Cordis Enterprise stent. AJNR Am J Neuroradiol 2005;26:1751-1756.

3. Lylyk P, Ferrario A, Pasbon B, Miranda C, Doroszuk G. Buenos Aires experience with the Neuroform self-expanding stent for the treatment of intracranial aneurysms. J Neurosurg 2005; 102:235-241.

4. Weber W, Bendszus M, Kis B, Boulanger T, Solymosi L, Kuhne D. A new self-expanding nitinol stent (Enterprise) for the treatment of wide-necked intracranial aneurysms: initial clinical and angiographic results in 31 aneurysms. Neuroradiology 2007;49:555-561.

5. Mocco J, Fargen KM, Albuquerque FC, Bendok BR, Boulos AS, Carpenter JS, et al. Delayed thrombosis or stenosis following enterprise-assisted stent-coiling: is it safe? Midterm results of the interstate collaboration of enterprise stent coiling. Neurosurgery 2011;69:908-913; discussion 913-904.

6. Heller R, Calnan DR, Lanfranchi M, Madan N, Malek AM. Incomplete stent apposition in Enterprise stent-mediated coiling of aneurysms: persistence over time and risk of delayed ischemic events. J Neurosurg 2013;118:1014-1022.

7. Heller RS, Miele WR, Do-Dai DD, Malek AM. Crescent sign on magnetic resonance angiography revealing incomplete stent apposition: correlation with diffusion-weighted changes in stent-mediated coil embolization of aneurysms. J Neurosurg 2011;115:624-632.

8. Heller RS, Malek AM. Parent vessel size and curvature strongly influence risk of incomplete stent apposition in enterprise intracranial aneurysm stent coiling. AJNR Am J Neuroradiol 2011;32:1714-1720.

9. Rossen JD, Chalouhi N, Wassef SN, Thomas J, Abel TJ, Jabbour PM, et al. Incidence of cerebral ischemic events after discontinuation of clopidogrel in patients with intracranial aneurysms treated with stent-assisted techniques. J Neurosurg 2012;117:929-933.

10. Yamada NK, Cross DT, Pilgram TK, Moran CJ, Derdeyn CP, Dacey RG. Effect of antiplatelet therapy on thromboembolic complications of elective coil embolization of cerebral aneurysms. AJNR Am J Neuroradiol 2007;28:1778-1782.

11. Fargen KM, Hoh BL, Welch BG, Pride GL, Lanzino G, Boulos AS, et al. Long-term results of enterprise stent-assisted coiling of cerebral aneurysms. Neurosurgery 2012;71:239-244; discussion 244.

12. Attizzani GF, Capodanno D, Ohno Y, Tamburino C. Mechanisms, pathophysiology, and clinical aspects of incomplete stent apposition.J Am Coll Cardiol 2014;63:1355-1367.

13. Foin N, Gutierrez-Chico JL, Nakatani S, Torii R, Bourantas $\mathrm{CV}$, Sen S, et al. Incomplete stent apposition causes high shear flow disturbances and delay in neointimal coverage as a function of strut to wall detachment distance: implications for the management of incomplete stent apposition. Circ Cardiovasc Interv 2014;7:180-189.

14. Kume T, Waseda K, Ako J, Sakata K, Yamasaki M, Shimohama $\mathrm{T}$, et al. Intravascular ultrasound assessment of postprocedural incomplete stent apposition. J Invasive Cardiol 2012;24:1316.

15. Moussa ID, Colombo A. Antiplatelet therapy discontinuation following drug-eluting stent placement: dangers, reasons, and management recommendations. Catheter Cardiovasc Interv 2009; 74:1047-1054.

16. Maeng M, Thim T, Chisholm G, Johansen M, Schmidt M, Sørensen HT, et al. TCT-55 Major Adverse Cardiac Events Associated With Discontinuation Of Clopidogrel Treatment Within The First Year After Coronary Stent Implantation In Western Denmark. Journal of the American College of Cardiology 2012;60:B17-B17.

17. Hwang G, Kim JG, Song KS, Lee YJ, Villavicencio JB, Setiawan NS, et al. Delayed Ischemic Stroke after Stent-assisted Coil Placement in Cerebral Aneurysm: Characteristics and Optimal Duration of Preventative Dual Antiplatelet Therapy. Radiology: Imaging Cancer 2014;273:194-201. 